\title{
Economia de pólen favorecida pela heteranteria em Desmocelis villosa (Melastomataceae)
}

\author{
Pollen economy enhanced by heteranthery in Desmocelis villosa (Melastomataceae)
}

\author{
Queroanne Isabel Xavier Ferreira ${ }^{1}$ \& Francielle Paulina de Araújo ${ }^{2,3}$
}

\begin{abstract}
Resumo
A heteranteria sempre despertou interesse quanto à sua funcionalidade. As flores de Desmocelis villosa (Melastomataceae), possuem estames dimorfos, sendo um grupo composto por cinco anteras amarelas e o outro por cinco anteras de cor púrpura. O presente estudo avaliou se a heteranteria promove divisão de trabalho nessa espécie. Foi realizado um experimento com remoção de anteras de cada tipo para se comparar a taxa de visitação por abelhas. Ao vibrar o conjunto de estames, as abelhas coletavam o pólen das anteras amarelas enquanto as de cor púrpura depositavam seu pólen na parte final do corpo das mesmas em uma posição próxima ao estigma favorecendo a polinização. Flores com anteras amarelas apresentaram maiores taxas de visitação que aquelas que tinham apenas anteras púrpuras. Nesta espécie a heteranteria se mostra como uma estratégia eficaz na economia do pólen utilizado com o serviço de polinização, pois embora os dois tipos de anteras apresentem alta porcentagem de grãos viáveis, as anteras amarelas apresentam significativamente menos grãos de pólen que as púrpuras, indicando que a planta estrategicamente reserva a maior parte do pólen para a reprodução sexuada. Palavras-chave: dimorfismo estaminal, divisão de trabalho, especialização funcional, polinização por vibração.
\end{abstract}

\begin{abstract}
Heteranthery has always aroused interest regarding its functionality. Desmocelis villosa (Melastomataceae) has flowers with dimorphic stamens, one group being composed of five yellow anthers and the other of five purple anthers. The current study assessed whether heteranthery promotes division of labor in this species. An experiment was performed with removal of anthers of each type in order to compare the visitation rate by bees. On vibrating the set of stamens, bees collected pollen from the yellow anthers, while the purple anthers deposited their pollen on the end part of their bodies in a position close to the stigma, thus allowing pollination. Flowers with yellow anthers showed higher visitation rates than those that solely had purple anthers. In this species, heteranthery is found to be an effective strategy for economizing pollen, which is used as a floral reward, given that, although the two types of anthers show a high percentage of viable grains, the yellow anthers have significantly fewer pollen grains than the purple ones, indicating that the plant strategically reserves most of the pollen for sexual reproduction purposes.
\end{abstract}

Key words: stamen dimorphism, division of labour, functional specialization, buzz pollination.

\section{Introdução}

Mais de 20.000 espécies de plantas apresentam apenas o pólen como recompensa para seus polinizadores, sendo conhecidas como flores de pólen (Vogel 1978). Muitas dessas plantas se adaptaram à polinização pelas abelhas, que dependem do pólen para alimentar suas larvas (Buchmann 1983; Michener 2007; Pohl et al. 2008). Assim, ter o pólen como única recompensa floral representa um dilema de pólen para a planta frente ao polinizador (Lunau et al. 2014), uma vez que o pólen coletado contêm os gametas masculinos da planta, fundamentais para sua reprodução sexuada, e ao mesmo tempo as abelhas são os vetores que transportam o pólen favorecendo a reprodução cruzada (Thorp 1979; Harder \& Thomson 1989; Westerkamp 2004b; Vallejo-Marín et al. 2009).

\footnotetext{
${ }^{1}$ Universidade Federal de Uberlândia, Inst. Biologia, Programa de Pós-graduação em Ecologia e Conservação dos Recursos Naturais, C.P. 593, 38400-902, Uberlândia, MG, Brasil.

${ }^{2}$ Universidade Estadual do Rio Grande do Sul, R. Assis Brasil 842, 95400-000, São Francisco de Paula, RS, Brasil.

${ }^{3}$ Autor para correspondência: fran.uergs@gmail.com
} 
Esta pressão seletiva fez com que muitas plantas desenvolvessem mecanismos para diminuir a perda excessiva do pólen para as abelhas (Westerkamp 2004a). Assim, a morfologia da flor afeta o comportamento dos polinizadores e a eficiência da transferência de pólen (Harder \& Barrett 1995; Williams 2007; Karron et al. 2009; Sandring \& Agren 2009), restringindo a quantidade de polinizadores capazes de realizar o trabalho com êxito. Exemplo desses mecanismos podem ser adaptações morfológicas como as quilhas ou lábios que escondem o pólen, ou ainda, anteras que só se abrem em poros apicais (Larson \& Barrett 1999; Westerkamp 2004b).

A maioria das espécies de angiospermas apresenta anteras que se abrem através de fendas longitudinais (Endress 1996), mas existe um grande número de espécies que apresenta anteras poricidas e a retirada do pólen das mesmas exige um comportamento específico das abelhas (Buchmann \& Hurley 1978). Neste caso, a polinização efetiva fica restrita apenas àquelas espécies que são capazes de produzir vibrações, ou "buzz pollination" (Buchmann 1983). No entanto, mesmo essas flores que apresentam anteras poricidas correm o risco de perder todo seu pólen para as abelhas que vibram podendo ficar sem polinização (Westerkamp 2004a; Castellanos et al. 2004). Assim, para evitar este problema, muitas plantas desenvolveram a heteranteria, ou seja, possuem dois tipos de estames em uma mesma flor que promove a divisão de trabalho, com um grupo de anteras destinado à polinização, e o outro grupo destinado à alimentação dos polinizadores (Müller 1883). Em geral, estas flores apresentam dois tipos de estames que diferem na posição, cor e/ou morfologia (Vogel 1978; Vallejo-Marín et al. 2009; Barrett 2010).

A heteranteria tem despertado interesse em sua funcionalidade desde a época de Charles Darwin e Fritz Müller (entre os anos 1883 a 1889) que acreditavam que os estames diferiam por apresentarem funções diferentes dentro da flor (Westerkamp 2004a,b; Luo et al. 2008). Existem algumas ideias sobre como ocorreria essa especialização funcional, uma delas é que os estames de alimentação seriam especialmente atraentes para as abelhas, como exemplo, apresentando uma cor chamativa e/ou localização acessível. Enquanto os estames para a polinização apresentariam coloração menos conspícua e uma localização mais próxima do estigma, fazendo com que o pólen se deposite em lugares estratégicos no corpo da abelha para a realização da polinização (Jesson \& Barret 2003; Vallejo-Marín et al. 2009). Deste modo, ocorreria uma deposição diferencial do pólen em regiões distintas do corpo das abelhas. O pólen das anteras de polinização ficaria localizado em uma parte do corpo das abelhas que não são alcançados durante a limpeza e, portanto, estaria destinado à polinização. Enquanto o pólen das anteras de alimentação ficaria localizado em partes do corpo de fácil alcance e seria usado para o provimento dos ninhos (Vallejo-Marín et al. 2010).

A heteranteria está presente em diversos grupos taxonômicos (Graham \& Barrett 1995; Jesson \& Barrett 2003) como exemplo, nas famílias Melastomataceae, Fabaceae, Bignoniaceae, Commelinaceae e Solanaceae. A família Melastomataceae é composta por cerca de 4.570 espécies distribuídas em cerca de 160 gêneros (Clausing \& Renner 2001) tendo a ocorrência de heteranteria fortemente difundida, ocorrendo em centenas de espécies e vários gêneros (VallejoMarín et al. 2010; Hoffmann \& Varasin 2011).

Apesar de ser um questionamento antigo, somente há pouco tempo Luo et al.(2008) comprovaram as ideias de Müller (1883) quanto à ocorrência da divisão de trabalho entre anteras dimorfas de uma espécie de Melastomataceae. Apesar de existir várias espécies desta família em diversas regiões, nenhum estudo com este enfoque foi realizado no Brasil até o momento. Sendo assim, o presente estudo teve como objetivo avaliar se a função do dimorfismo estaminal em Desmocelis villosa Aubl. Naud (Melastomataceae) é promover a divisão de trabalho.

\section{Material e Métodos}

Área de estudo

$\mathrm{O}$ presente estudo foi realizado em uma área de vereda na Reserva Ecológica do Clube Caça e Pesca Itororó de Uberlândia (CCPIU), Uberlândia, MG, Brasil. Essa reserva está situada a oeste do município, distando $8 \mathrm{~km}$ do centro da cidade nas coordenadas geográficas $18^{\circ} 55^{\prime} 23^{\prime \prime} \mathrm{S}$ e 48 17'19"W (Lima et al. 1989). O clima da região é caracterizado por duas estações, sendo uma seca e fria entre os meses de maio a setembro e outra chuvosa e quente que vai de outubro a abril. $\mathrm{Na}$ classificação de Köppen, o clima pode ser definido como Aw, tendo ocorrências de altas temperaturas no verão (acima de $35^{\circ} \mathrm{C}$ ) e geadas ocasionais no inverno (Rosa et al. 1991). 
Espécie estudada

Desmocelis villosa Aubl. Naud (Melastomataceae) (Fig. 1) tem hábito herbáceo ou subarbustivo, podendo atingir até $1,5 \mathrm{~m}$ de altura, possui ramos altamente vilosos, de fácil reconhecimento no campo. Suas flores são pentâmeras, as pétalas possuem coloração rósea, possui 10 estames dimorfos (Fig. 1a), sendo que metade deles é menor com conectivos curtos e auriculados na base, com anteras amarelas, curtas e espessas. A outra metade, localizada próxima ao estigma, é maior de coloração púrpura com conectivos longos e dois apêndices lineares mais longos na base da antera, que é rostelada. A espécie é geralmente encontrada em solo brejoso, arenoso, ou entre rochas, sempre próximas a locais úmidos. Distribui-se nas Guianas, Colômbia, Venezuela, Bolívia e Brasil. No Brasil está presente nos estados do Rio de Janeiro, Minas Gerais, Distrito Federal, Mato Grosso do Sul, Mato Grosso, Bahia, Piauí, Pará, Amazonas e Roraima, com ocorrência no Cerrado (Cogniaux 1885; Mendonza \& Ramírez 2006). Para avaliar a função da heteranteria em $D$. villosa, foram feitas observações e experimentos no campo durante o período de abril a junho de 2011 e de janeiro a junho de 2012 .

\section{Comportamento dos visitantes florais}

Para avaliar o comportamento dos visitantes florais, foram realizadas observações focais em diferentes indivíduos. Foram feitas um total de 30 horas de observação, distribuídas no período manhã e tarde. Após a determinação do horário de maior visitação de polinizadores, as observações foram concentradas no período da manhã entre $9 \mathrm{~h}$ e $10 \mathrm{~h}$. Um indivíduo de cada espécie de abelha que visitou D. villosa foi capturado com rede entomológica para posterior identificação.

\section{Desenho experimental}

Neste estudo foi utilizado um desenho experimental adaptado de Luo et al. (2008). Para tanto, foram utilizados dez indivíduos escolhidos ao acaso, que tivessem pelo menos doze flores cada. As flores que excediam o conjunto foram removidas para não interferirem na atratividade do indivíduo. Nesses indivíduos as flores foram manipuladas, sendo utilizadas quatro dessas para cada tratamento: 1) flores deixadas intactas (controle); 2) anteras púrpuras foram removidas; 3 ) anteras amarelas foram removidas (Fig. 1a-d). As observações e os tratamentos foram conduzidos utilizando uma planta focal por dia e tomou-se o cuidado de realizar as observações somente em dias ensolarados sempre nos mesmos horários. Após a manipulação das flores foi registrado o comportamento dos visitantes em seções de observação de uma hora contínua (entre 9 h e 10 h). Para cada tratamento foi registrado o número de visitas/flor/hora. A temperatura média e umidade relativa foram registradas para todos os dias de observação.

Quantificação de pólen nas anteras antes e após as atividades de coleta das abelhas

Para estimar a quantidade total de pólen presente e que permanecia nos dois grupos de anteras no final do período de visitação, foram coletados 10 botões florais em pré-antese de cinco indivíduos e outras 10 flores abertas por volta das $11 \mathrm{~h} 00 \mathrm{~min}$. Todo material coletado foi fixado em FAA 50 (Johansen 1940). No laboratório, foi selecionada uma antera de alimentação e outra de polinização para cada botão ou flor e as mesmas foram esmagadas separadamente em eppendorfs contendo uma solução de glicerina e carmim acético (proporção de 1:3). A solução foi agitada em vórtex por 60 segundos e em seguida foram retiradas 10 amostras de $1 \mu \mathrm{L}$ da suspensão de cada tipo de antera com uma micropipeta calibrada. As amostras foram transferidas para lâminas e em todas foram quantificados os grãos de pólen sob um microscópio. Por fim, os valores foram multiplicados pelo fator de diluição, para assim obter uma estimativa do número de grãos de pólen por antera e para os dois grupos de estames (ver Luo et al. 2008).

\section{Viabilidade polínica}

Para avaliar se os grãos de pólen diferem quanto à viabilidade de acordo com o tipo de antera de origem (púrpura ou amarela), botões em préantese foram ensacados em cinco indivíduos e suas flores recém-abertas foram coletadas e mantidas em espuma vegetal hidratada. No laboratório, os grãos de pólen de cada grupo de estames foram retirados e mantidos em lâminas diferentes. Os mesmos foram submetidos ao teste DAB (3.3' Diaminobenzidina, Sigma FastTM), que detecta a presença de peroxidases no citoplasma (Dafni et al. 2005). Cada lâmina foi fotografada e os 100 primeiros grãos de pólen visualizados foram registrados quanto à proporção de viáveis e não viáveis, utilizando o programa Anati Quanti (Aguiar et al. 2007). 

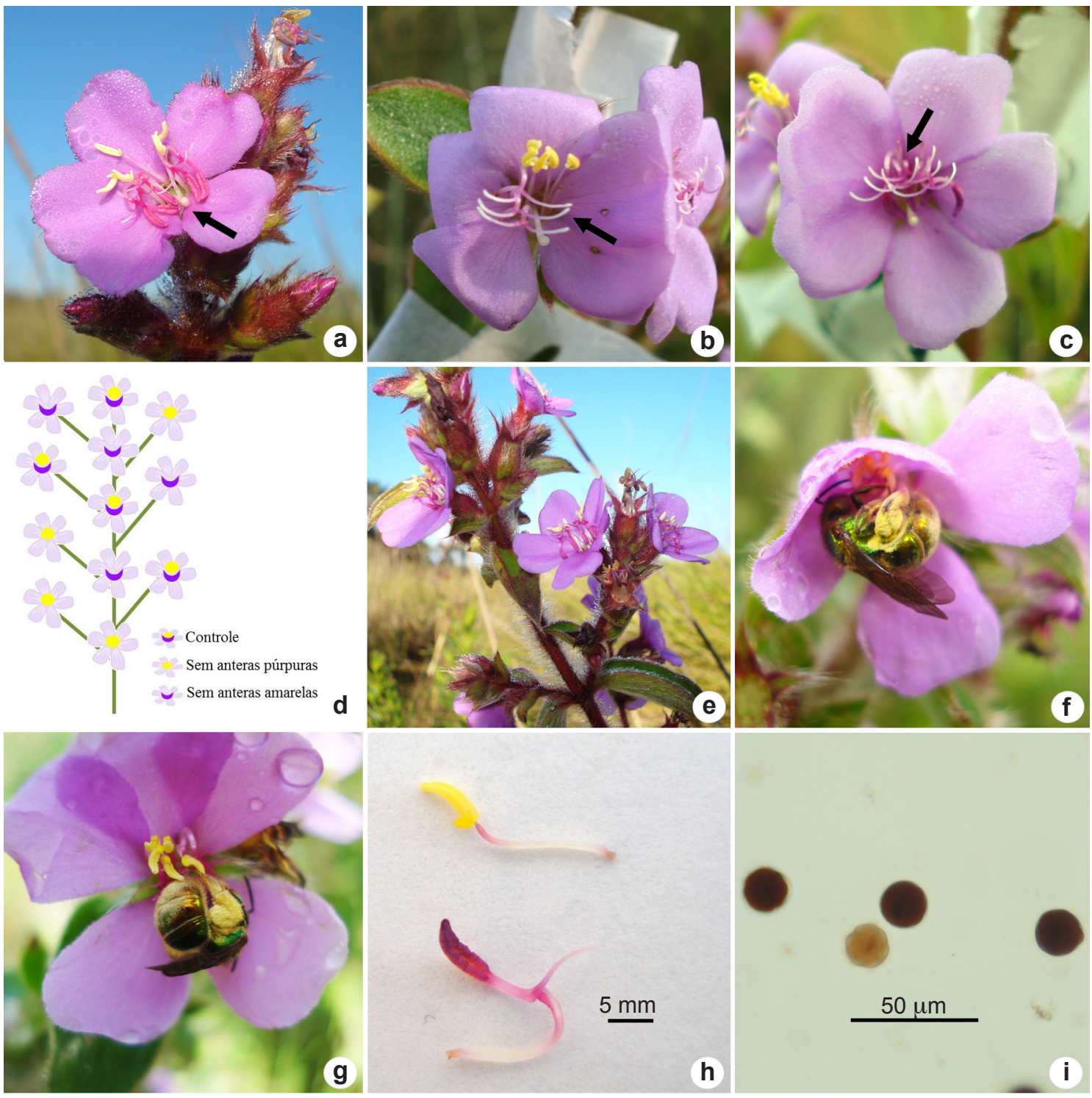

Figura 1 - Desenho experimental e características gerais de Desmocelis villosa (Melastomataceae) - a-c. tratamentos utilizados no experimento - a. flor com estames dimorfos (essa flor intacta representa o controle, e a seta indica o estigma localizado próximo aos estames de cor púrpura); b. flor sem as anteras púrpuras; c. flor sem anteras amarelas (setas em ' $b$ ' e ' $c$ ' indicam os locais de onde as anteras foram removidas para cada tratamento). d. esquema representando o desenho experimental, onde cada bloco consistiu em um indivíduo com 12 flores, sendo utilizadas quatro dessas para cada tratamento. e. ramo florido. f. abelha do gênero Augochloropsis coletando pólen das anteras púrpuras, e o mesmo indivíduo em g. coletando pólen das anteras amarelas. h. estames com suas respectivas anteras: amarela acima e púrpura abaixo. i. grãos de pólen provenientes de anteras púrpuras corados com DAB (3.3' diaminobenzidina, Sigma FastTM), os grãos escuros são viáveis e os claros são inviáveis.

Figure 1 - Experimental design and general characteristics of Desmocelis villosa (Melastomataceae) - a-c. treatments used in the experiment - a. flower with two sets of stamen (this flower represents the control, and the arrow indicates the stigma located close to the purple anthers); b. flower without the purple anthers; c. flower without the yellow anthers (arrows in 'b' and 'c' indicates the place where the anthers were removed for each treatment). d. scheme showing the experimental design, where each bloc consisted of an individual with 12 flowers, being used four of these for each treatment. e. flowery branch. f. Augochloropsis bee collecting pollen in the yellow anthers, and the same individual in g. collecting pollen in the purple anthers. h. stamens with its yellow and purple anthers. i. pollen grains from the purple anthers stained with DAB (3.3 'diaminobenzidine, Sigma FastTM), dark grains are viable and non-viable are clear. 


\section{Análise estatística}

Foram usados testes t-student para comparar as diferenças na quantidade de pólen entre anteras púrpuras e amarelas e as proporções de pólen que sobraram após a visitação dos polinizadores. Para testar as diferenças nas frequências de visitas entre as flores dos diferentes tratamentos foi utilizada uma análise ANOVA. Os pacotes estatísticos utilizados foram o SPSS para análise das pressuposições e SISVAR para análise dos testes de médias.

\section{Resultados}

Comportamento dos visitantes florais

As flores de $D$. villosa iniciam a abertura de manhã entre $7 \mathrm{~h}$ e $8 \mathrm{~h}$ e duram menos de um dia, entrando em senescência a partir das $13 \mathrm{~h}$. No período de estudo receberam visitas de abelhas por volta das $8 \mathrm{~h}$ e $12 \mathrm{~h}$. Foram registradas cinco espécies de abelhas (Tab. 1) visitando as flores e todas apresentaram comportamento de vibração - "buzz pollination".

As abelhas de médio e grande porte visitaram a flores de $D$. villosa pousando nas pétalas, sempre direcionadas para o conjunto de anteras amarelas. Ao emitir vibrações, os dois grupos de anteras liberavam pólen diretamente no corpo da abelha. Não foi possível diferenciar, no corpo das abelhas, o local de deposição de cada tipo de pólen, no entanto, devido à arquitetura floral os dois tipos de anteras depositam seus respectivos grãos de pólen em locais diferentes. O pólen proveniente das anteras púrpuras fixa-se principalmente no final do abdômen, local que tem contato com o estigma das flores ficando este destinado à polinização (Fig. 1a). Já as anteras amarelas que são as mais conspícuas provavelmente liberam seu pólen em locais do corpo das abelhas onde é mais facilmente coletado e posteriormente utilizado para a alimentação. Entretanto, as abelhas de pequeno porte (Tab.1) do gênero Augochloropsis pousavam nas anteras coletando pólen separadamente, primeiro das amarelas, depois das púrpuras (Fig. 1f,g).

Nos testes para avaliar a taxa de visitação em flores com a remoção de um conjunto de anteras (púrpuras ou amarelas) foi observado que o número de visitas em flores controle $(4,10$ $\pm 2,01$ visitas $\left.\mathrm{h}^{-1}, \mathrm{n}=10\right)$ e flores com apenas anteras amarelas $\left(3,50 \pm 2,2\right.$ visitas $\left.\mathrm{h}^{-1}, \mathrm{n}=10\right)$ não diferiram (ver Tab. 2). Porém, nas flores em que foram mantidas apenas as anteras púrpuras o número de visitas $\left(1,90 \pm 1,2\right.$ visitas $\left.\mathrm{h}^{-1}, \mathrm{n}=10\right)$ foi inferior ao dos demais tratamentos (Tab. 2).

\section{Diferenças do pólen nos dois tipos} de anteras

As anteras púrpuras antes das visitas das abelhas apresentaram significativamente mais grãos de pólen que as anteras amarelas 128.800 \pm 30.546 contra $46.400 \pm 3.565$, respectivamente, ( $t=8,47 ; \mathrm{P}<0,001 ; \mathrm{N}=10$ flores $)$.

A quantidade de grãos de pólen estimada após as atividades das abelhas foi em média $80.400 \pm 29.767(\mathrm{~N}=10$ flores $)$ nas anteras púrpuras e $9.150 \pm 4.384(\mathrm{~N}=10$ flores $)$ nas anteras amarelas. Comparando os valores estimados antes e depois das atividades das abelhas, menos de $38 \%$ do pólen proveniente das anteras púrpuras foi removido pelas abelhas,

Tabela 1 - Visitantes florais de Desmocelis villosa (Melastomataceae) e estames escolhidos para a coleta de pólen. Table 1 - Floral visitors of Desmocelis villosa (Melastomataceae) and stamens chosen for pollen collection.

\begin{tabular}{lcc}
\hline Família / Espécies de abelhas & $\begin{array}{c}\text { Estames de polinização } \\
\text { (anteras púrpuras) }\end{array}$ & $\begin{array}{c}\text { Estames de alimentação } \\
\text { (anteras amarelas) }\end{array}$ \\
\hline Apidae & & $\mathrm{x}$ \\
Eulaema nigrita Lepeletier & $\mathrm{x}$ \\
Euglossa cordata L. & $\mathrm{x}$ \\
Euglossa fimbriata Rebêlo \& Moure & $\mathrm{x}$ & $\mathrm{x}$ \\
Halictidae & $\mathrm{x}$ & $\mathrm{x}$ \\
Augochloropsis rotalis Vachal & & \\
Augochloropsis cyanea Schrottky & & \\
\hline
\end{tabular}


Tabela 2 - Frequência de visitas de abelhas (média do número de visitas/flor/hora) às flores de Desmocelis villosa (Melastomataceae) na Reserva Ecológica do Clube Caça e Pesca Itororó de Uberlândia, MG, Brasil.

Table 2 - Frequency of bee visits (average of number of visits/flower/hour) to flowers of Desmocelis villosa (Melastomataceae) in Ecological Reserve of Clube Caça e Pesca Itororó de Uberlândia, MG, Brazil.

\begin{tabular}{cc}
\hline Presença de estames & $\overline{\boldsymbol{X}}_{\text {Número de visitas h }} \mathbf{c}_{\mathbf{S}} \mathbf{S D}$ \\
\hline Alimentação e Polinização (controle) & $4,10 \pm 2,01$ a \\
Somente alimentação & $3,50 \pm 2,2 \mathrm{a}$ \\
Somente polinização & $1,90 \pm 1,2 \mathrm{~b}$ \\
\hline
\end{tabular}

\begin{tabular}{ll}
\hline $\mathrm{W}(\mathrm{P}): 0,969(0,524)$ & CV: $37,56 \%$ \\
${ }^{1} \mathrm{~F}(\mathrm{P}): 1,940(0,163)$ & DMS: 1,36 \\
${ }^{2} \mathrm{~F}(\mathrm{P}): 9,141(0,002)$ &
\end{tabular}

Médias \pm desvio padrão seguidas por letras distintas, na coluna, diferem pelo teste de Tukey à 0,05 de significância; W(P): Estatística do teste de Shapiro-Wilk $(\mathrm{P}>0,05) ;{ }^{1} \mathrm{~F}(\mathrm{P})$ : Teste de Levene $(\mathrm{P}>0,05) ;{ }^{2} \mathrm{~F}(\mathrm{P})$ : Teste de Snedecor (ANOVA; $\left.\mathrm{P}<0,05\right), \mathrm{N}=10$.

Average \pm standard deviation followed by different letters in the column, differ by Tukey test at 0.05 significance; W(P): Statistics of Shapiro-Wilk $(\mathrm{P}>0,05) ;{ }^{1}$ $\mathrm{F}(\mathrm{P})$ : Levene tests $(\mathrm{P}>0,05) ;{ }^{2} \mathrm{~F}(\mathrm{P})$ : Snedecor tests (ANOVA; $\left.\mathrm{P}<0,05\right), \mathrm{N}=10$.

contra $80 \%$ dos removidos das anteras amarelas. Portanto, houve uma remoção diferencial sendo retirado proporcionalmente mais pólen das anteras amarelas. Em geral os estames de D. villosa apresentaram alta porcentagem de viabilidade polínica nos dois tipos de anteras (Fig. 1h,i). A média da porcentagem de grãos de pólen viáveis nas anteras púrpuras foi de $96,5 \pm 3,3$ ( $\mathrm{n}=5$ flores) e nas amarelas foi de $95,7 \pm 6,1$ ( $\mathrm{n}=5$ flores).

\section{Discussão}

Este estudo de caráter experimental foi o primeiro feito no Brasil testando a hipótese de divisão de trabalho. Os dados apoiam os resultados de Luo et al. (2008), confirmando as ideias iniciais de Müller (1883). Em D. villosa, assim como nas espécies estudadas até o momento, existe um conjunto de anteras mais conspícuo responsável pela atração e alimentação dos visitantes e outro menos conspícuo localizado estrategicamente em um local que favoreça a polinização cruzada (ver Jesson \& Barret 2003; Vallejo-Marín et al. 2009). Existem diferenças entre os dois tipos de anteras tanto no tamanho quanto na posição dentro da flor e as cores dessas estruturas ajudam na função de cada grupo. A cor amarela torna as anteras mais visíveis enquanto a cor púrpura camufla as anteras ajudando a direcionar o pólen para a polinização (Lunal 2000; Heuschen et al. 2005).

A heteranteria surge quando os polinizadores consomem mais pólen do que a planta pode fornecer de forma otimizada em troca dos serviços de polinização (Vallejo-Marín et al. 2009). Assim, muitas plantas evoluíram em direção à heteranteria para poupar parte do pólen para a reprodução sexuada (Westerkamp 1996). Desta forma, comparada às demais flores de pólen, há indícios de que $D$. villosa apresenta vantagens em termos de economia desse recurso, pois além de limitar o número de anteras para a alimentação das abelhas, ou seja, apenas $50 \%$ do total, essa espécie disponibiliza menos pólen nas anteras de alimentação comparado às de polinização. Outros estudos também registraram menor quantidade de pólen nas anteras de alimentação (ver Luo et al. 2008; Vallejo-Marín et al. 2009; Brito \& Sazima 2012).

Avaliando as possibilidades evolutivas para a especialização funcional entre os diferentes tipos de estames com finalidade de economizar pólen, as flores que ofertam néctar poderiam levar à evolução de anteras com pouco ou nenhum pólen (Vogel 1978). Contudo, em flores que só ofertam pólen isso seria muito difícil de acontecer, pois as abelhas exercem grande pressão seletiva, uma vez que conseguem perceber a quantidade de pólen liberada pelas anteras durante a vibração e aprendem a evitar aquelas que não têm pólen (Buchmann \& Cane 1989; Harder 1990; Luo et al. 2008).

Darwin acreditava que a divisão de trabalho levaria à evolução de pólen estéril nos estames de alimentação (Luo et al. 2008). Lloyd (2000) 
também interpretou que a heteranteria poderia permitir a esterilização de parte dos gametas para beneficiar os gametas restantes destinados à polinização. Em outro estudo considerando algumas espécies de Commelinaceae com heteranteria foi observado polimorfismo polínico com uma mistura de grãos de pólen grandes e pequenos em igual proporção para todos os tipos de anteras, porém com alta taxa de esterilidade nos grãos pequenos (Hrycan \& Davis 2005). Ao contrário dos resultados de Luo et al. (2008) e Hrycan \& Davis (2005), D. villosa apresentou alta porcentagem de pólen viável nos dois tipos de anteras. Podendo significar que apesar de ter reduzido a quantidade de pólen de alimentação, essa espécie ainda não reduziu os custos com o tipo de pólen ofertado aos polinizadores. Além dos dados de viabilidade polínica, seria importante realizar testes de polinização para avaliar o potencial reprodutivo do pólen dos dois tipos de anteras.

Apesar da ocorrência de abelhas de pequeno porte coletando pólen nos dois tipos de anteras em $D$. villosa, essas provavelmente não exercem grande impacto, pois ao final do período de atividade da comunidade de abelhas, ainda sobra proporcionalmente mais pólen nas anteras de polinização que nas anteras de alimentação. Provavelmente esse fato ocorre porque abelhas de pequeno porte não são capazes de extrair eficientemente grandes quantidades de pólen (Snow \& Roubik 1987; Vallejo-Marín et al. 2009), além do fato de existir mais pólen nas anteras de polinização.

Portanto, de uma forma geral, em $D$. villosa a estratégia de dividir o pólen em dois tipos de estames resolve o dilema entre abelha e flor, mantendo a relação estável (Luo et al. 2008) e ainda se mostra como um mecanismo eficaz na economia de pólen, uma vez que as anteras de alimentação produzem menos pólen que as anteras de polinização, como constatado para outras espécies com heteranteria (Hrycan \& Davis 2005). Além disso, nessa espécie, as abelhas removem diferencialmente mais pólen das anteras amarelas. Isso indica que provavelmente a espécie desenvolveu estratégias para dosar a quantidade de pólen que é liberada durante as visitas das abelhas, direcionando a maior parte desse recurso para a reprodução sexuada. Seria importante estudar outras espécies de Melastomataceae que apresentam diferentes graus de dimorfismo estaminal para saber o quanto essas estruturas precisam se diferir para que haja divisão de trabalho.

\section{Agradecimentos}

Ao Dr. João Paulo Ribeiro Oliveira, o auxílio na estatística. Às pesquisadoras Sheila Teodoro, Sarah Oliveira e Larissa Araújo, a assistência no trabalho de campo. À Dra. Daniela Simão, as sugestões na elaboração do manuscrito. Ao Mestre Thiago H. Tosta, a identificação das abelhas. Ao amigo John Upson, a ajuda com o abstract. À amiga Alessandra Rosa Oliveira, a revisão do texto. À Capes-PRODOC, o apoio financeiro.

\section{Referências}

Aguiar, T.V.; Sant'ana-Santos, B.F.; Azevedo, A.A. \& Ferreira, R.S. 2007. Anati Quanti: software de análises quantitativas para estudos em anatomia vegetal. Planta Daninha 25: 649-659.

Barrett, S.C.H. 2010. Darwin's legacy: the forms, function and sexual diversity of flowers. Philosophical Transactions of the Royal Society B 365: 351-368.

Brito, V.L.G. \& Sazima M. 2012. Tibouchina pulchra (Melastomataceae): reproductive biology of a tree species at two sites of an elevational gradient in the Atlantic rainforest in Brazil. Plant Systematics and Evolution 298: 1271-1279.

Buchmann, S.L \& Cane, J.H. 1989. Bees assess pollen returns while sonicating Solanum flowers. Oecologia 81: 289-294.

Buchmann, S.L. \& Hurley, J.P. 1978. A biophysical model for buzz pollination in Angiosperms. Journal of Theoretical Biology 72: 639-657.

Buchmann, S.L. 1983. Buzz pollination in angiosperms. In: Jones, C.E. \& Little, R.J. (eds.). Handbook of experimental pollination biology. Van Nostrand Reinhold, New York. Pp. 73-113.

Castellanos, M.C.; Wilson, P. \& Thomson, J.D. 2004. 'Anti-bee' and 'pro-bird' changes during the evolution of hummingbird pollination in Penstemon flowers. Journal of Evolutionary Biology 17: 876885.

Clausing, G. \& Renner, S.S. 2001. Molecular phylogenetics of Melastomataceae and Memecylaceae: implications for character evolution. American Journal of Botany 88: 486-498.

Cogniaux, C.A. 1885. Melastomataceae - Tibouchineae. In: von Martius, C.F.P. \& Eichler, A.W. (eds.). Flora Brasiliensis. Frid. Fleischer, Lipsae, Munchen. Vol. 14, part 3, pp 233-234.

Dafni, A.; Kevan, P.G. \& Husband, B.C. 2005. Practical pollination biology. Enviroquests, Ltd. Cambridge, Ontario. 590p.

Endress, P.K. 1996. Diversity and evolutionary trends in angiosperm anthers. In: D'Arcy, W.G. \& Keating, R.C. (eds.). The anther: form, function and physiology. Cambrige University Press, Cambrige. Pp. 92-110. 
Fracasso, C.M. \& Sazima, M. 2004. Polinização de Cambessedesia hilariana (Kunth) DC. (Melastomataceae): sucesso reprodutivo versus diversidade, comportamento e freqüência de visitas de abelhas. Revista Brasileira de Botânica 24: 797-804.

Graham, S.W. \& Barrett S.C.H. 1995. Phylogenetic systematics of Pontederiales: implications for breeding-system evolution. In: Rudall, P.J.; Cribb, P.J.; Cutler, D.F. \& Humphries, C.J., (eds.). Monocotyledons: systematics and evolution. Royal Botanical Gardens, Kew. Pp. 415-441.

Harder, L.D. \& Thomson, J.D. 1989. Evolutionary options for maximizing pollen dispersal of animal-pollinated plants. The American Naturalist 133: 323-344.

Harder, L.D. \& Barrett, S.C.H. 1995. Mating cost of large floral displays in hermaphrodite plants. Nature 373: 512-515.

Harder, L.D. 1990. Behavioral responses by bumble bees to variation in pollen availability. Oecologia 85: 41-47.

Heuschen, B.; Gumbert, A. \& Lunau, K. 2005. A generalized mimicry system involving angiosperm flower colour, pollen and bumblebees' inate colour preferences. Plant Systematics and Evolution 252: 121-137.

Hrycan, W.C. \& Davis, A.R. 2005. Comparative structure and pollen production of the stamens and pollinatordeceptive staminodes of Commelina coelestis and C. dianthifolia (Commelinaceae). Annals of Botany 95: 1113-1130.

Hoffmann, G.M. \& Varassin, I.G. 2011. Variação da viabilidade polínica em Tibouchina (Melastomataceae). Rodriguésia 62: 223-228.

Jesson, L.K. \& Barrett, S.C.H. 2003. The comparative biology of mirror-image flowers. International Journal of Plant Sciences 164: S237-S249.

Karron, J.D.; Holmquist, K.G.; Flanagan, R.J. \& Mitchell, R.J. 2009. Pollinator visitation patterns strongly influence among-flower variation in selfing rate. Annals of Botany 103: 1379-1383.

Larson, B.M.H. \& Barrett, S.C.H. 1999. The pollination ecology of buzz pollinated Rhexia virginica (Melastomataceae). American Journal of Botany 86: 502-511.

Lima, S.C.; Rosa, R. \& Feltran-Filho, A. 1989. Mapeamento do uso do solo no município de Uberlândia-MG, através de imagens TM/LANDSAT. Sociedade \& Natureza 1: 127-145.

Lloyd, D.G. 2000. The selection of social actions in families. III. Teproducctively disabled individuals and organs. Evolutionary Ecology Research 2: 29-40.

Lunal, T.D.K. 2000. The ecology and evolution of visual pollen signals. Plant Systematics and Evolution 222: 89-111.
Lunau, K.; Piorek V.; Krohn, O. \& Pacini, E. 2014. Just spines-mechanical defense of malvaceous pollen against collection by corbiculate bees. Apidologie 46: 144-149.

Luo, Z.; Zhang, D. \& Renner, S.S. 2008. Why two kinds of stamens in buzz-pollinated flowers? Experimental support for Darwin's division-of-labour hypothesis. Functional Ecology 22: 794-800.

Mendoza, H. \& Ramírez, B. 2006. Guía ilustrada de géneros de Melastomataceae y Memecylaceae de Colombia. Instituto de Investigación de Recursos Biológicos Alexander Von Humboldt, Universidad de Cauca, Bogotá D.C. 288p.

Michener, C.D. 2007. The bees of the world, $2^{\text {nd }}$ ed. John Hopkins University Press, Baltimore. 953p. Müller, F. 1883. Two kinds of stamens with different functions in the same flower. Nature 27: 364-365.

Nimer, E. \& Brandão, A.M.P.M. 1989. Balanço hídrico e clima da região dos cerrados. IBGE, Rio de Janeiro. 121p.

Pohl, M.; Watolla, T. \& Lunau, K. 2008. Anthermimicking floral guides exploit a conflict between innate preference and learning in bumblebees (Bombus terrestris).Behavioral Ecology and Sociobiology 63: 295-302.

Renner, S.S. 1989. A survey of reproductive biology in neotropical Melastomataceae and Memecylaceae. Annals of the Missouri Botanical Garden 76: 496-518.

Rosa, R.; Lima S.C. \& Assunção W.L. 1991. Abordagem preliminar das condições climáticas de UberlândiaMG. Sociedade e Natureza 3: 91-108.

Sandring, S. \& Agren, J. 2009. Pollinator-mediated selection on floral display and flowering time in the perennial herb. Arabidopsis lyrata. Evolution 63: 1292-1300.

Snow, A.A. \& Roubik, D.W. 1987. Pollen deposition and removal by bees visiting two tree species in Panama. Biotropica 19: 57-63.

Thorp, R.W. 1979. Structural, behavioral, and physiological adaptations of bees (Apoidea) for collecting pollen. Annals of Missouri Botanical Gardens 66: 788-812.

Vallejo-Marín, M.; Manson, J.S.; Thomson, J.D. \& Barrett, S.C.H. 2009. Division of labour within flowers: heteranthery, a floral strategy to reconcile contrasting pollen fates. Journal of Evolutionary Biology 22: 828-839.

Vallejo-Marín, M.; da Silva, E.M.; Sargent, R.D. \& Barret, S.C.H. 2010. Trait correlates and functional significance of heteranthery in flowering plants. New Phytologist 188: 418-425.

Vogel, S. 1978. Evolutionary shifts from reward to deception in pollen flowers. In: Richards, A.J. (ed.). The pollination of flowers by insects. Academic Press, London. Pp. 89-96. 
Westerkamp, C. 1996. Pollen in bee-flower relations. Some considerations on melittophily. Botanica Acta 109: 325-332.

Westerkamp, C. 2004a. Flores e abelhas na disputa. Ciência Hoje 34: 66-68.

Westerkamp, C. 2004b. Ricochet pollination in Cassias and how bees explain enantiostyly. In: Freitas, B.M.
\& Pereira, J.O.P. (eds.). Solitary bees. Conservation, rearing and management for pollination. Imprensa universitária, Fortaleza. Pp.225-230.

Williams, C.F. 2007. Effects of floral display size and biparental inbreeding on outcrossing rates in Delphinium barbeyi (Ranunculaceae). American Journal of Botany 94: 1696-1705. 